\title{
Tattoo-like epidermal electronics as skin sensors for human-machine interfaces
}

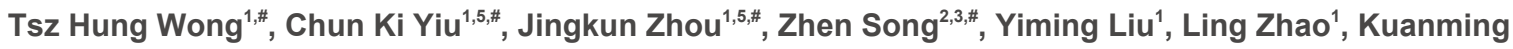 \\ $\mathrm{Yao}^{1}$, Wooyoung Park ${ }^{1}$, Woojung Yoo' ${ }^{1}$ Enming Song ${ }^{4}$, Zhaoqian $\mathrm{Xie}^{2,3}$, Xinge $\mathrm{Yu}^{1,5}$ \\ 'Department of Biomedical Engineering, City University of Hong Kong, Hong Kong 999077, China. \\ ${ }^{2}$ State Key Laboratory of Structural Analysis for Industrial Equipment, Department of Engineering Mechanics, Dalian University \\ of Technology, Dalian 116024, Liaoning, China. \\ ${ }^{3}$ Ningbo Institute of Dalian University of Technology, Ningbo 315016, Zhejiang, China. \\ ${ }^{4}$ Institute of Optoelectronics, Fudan University, Shanghai 200438, China. \\ ${ }^{5}$ Hong Kong Centre for Cerebro-Cardiovascular Health Engineering, Hong Kong 999077, China. \\ \#Authors contributed equally.
}

Correspondence to: Prof. Enming Song, Shanghai Frontiers Science Research Base of Intelligent Optoelectronics and Perception, Institute of Optoelectronics, Fudan University, 220 Handan Road, Shanghai 200433, China. E-mail: sem@fudan.edu.cn; Prof. Zhaoqian Xie, State Key Laboratory of Structural Analysis for Industrial Equipment, Department of Engineering Mechanics, Dalian University of Technology, No.2 Linggong Road, Ganjingzi District, Dalian 116024, Liaoning, China. E-mail: zxie@dlut.edu.cn; Xinge Yu, Department of Biomedical Engineering, City University of Hong Kong, 83 Tat Chee Avenue, Kowloon Tong, Hong Kong 999077, China. E-mail: xingeyu@cityu.edu.hk

How to cite this article: Wong TH, Yiu CK, Zhou J, Song Z, Liu Y, Zhao L, Yao K, Park W, Yoo W, Song E, Xie Z, Yu X. Tattoo-like epidermal electronics as skin sensors for human-machine interfaces. Soft Sci 2021;1:10. https://dx.doi.org/10.20517/ss.2021.09

Received: 30 Jul 2021 First Decision: 10 Aug 2021 Accepted: 23 Sep 2021 First online: 23 Sep 2021 Published: 13 Oct 2021

Academic Editor: Zhifeng Ren Copy Editor: Yue-Yue Zhang Production Editor: Yue-Yue Zhang

\begin{abstract}
Flexible electronic skin (e-skin) has been successfully utilized in diverse applications, including prosthesis sensing, body-motion monitoring and human-machine interfaces, due to its excellent mechanical properties and electrical characteristics. However, current e-skins are still relatively thick (> $10 \mu \mathrm{m})$ and uncomfortable for long-term usage on the human body. Herein, an ultrathin skin-integrated strain sensor with miniaturized dimensions, based on the piezoresistive effect, with excellent stability and robustness, is introduced. The fractal curve-shaped Au electrode in a serpentine format, which is the dominant component of the strain sensor, is sensitive to ambient strain variations and can turn the mechanical motion into a stable electrical signal output. With the advanced design of metallic electrodes, the device presents good operational stability and excellent mechanical tolerance towards bending, stretching and twisting. The stain sensor allows intimate mounting onto the human epidermal surface for the detection of body motion. By adopting a liquid bandage as an encapsulation layer, the device exhibits an ultrathin thickness $(6.2 \mu \mathrm{m})$, high sensitivity towards mechanical deformations and capability for the clear
\end{abstract}


detection of motion, such as walking, finger bending and the human pulse rate with identifiable electrical signals. Furthermore, the tattoo-like strain sensor is applied in robotic control by tracing finger bending motion and results in the smooth control of a robotic hand nearly without any detention. This e-skin design exhibits excellent potential for wearable electronics and human-machine interfaces.

Keywords: Skin-integrated electronics, ultrathin, excellent repeatability, fractal design, stretchable electronics

\section{INTRODUCTION}

Recently, epidermal electronics have attracted significant attention globally as a result of their excellent mechanical properties and electronic characteristics ${ }^{[1-10]}$. Until now, extensive flexible strain sensors have been developed based on various operational principles, such as piezoelectricity ${ }^{[1-14]}$, triboelectricity ${ }^{[15-17]}$, piezoresistivity ${ }^{[18-21,22]}$. Among the reported strain sensors, piezoelectric and triboelectric devices have raised extremely high interests for their natural characteristics in self-powering ${ }^{[23-31]}$. However, the instantaneous electrical signals and fast attenuation of electrical performance significantly limit their further development in the application of electronic skin (e-skin) ${ }^{[32]}$. Distinguished from self-powered strain sensors, e-skin, based on the piezoresistive effect, provides functions closer to human skin, capable of the accurate real-time detection of strain variation ${ }^{[33-38]}$. The resistance change of the piezoresistivity in e-skin can be conveniently transformed into voltage changes via real-time electrical monitoring of the sensor, which is then recognized by a microcontroller for later robotic control.

Benefitting from the fast development of materials science, many piezoresistive effect-based e-skins have been reported by incorporating advanced conductive materials, such as graphene and polydimethylsiloxane (PDMS) composites ${ }^{[39-42]}$, ion-conducting hydrogels ${ }^{[43-45]}$ and graphene-coated fabrics ${ }^{[46-48]}$. To achieve sufficient repeatability and stability, the fabrication process of newly-developed materials tends to require expensive equipment and rigorous experimental environments, which are not readily available with standard facilities in academic cleanrooms. Compared to these strain sensors, e-skins based on metal electrodes can realize low-cost fabrication, as well as high stability and repeatability with sufficient electrical sensitivity ${ }^{[4]}$. As a noble metal, gold $(\mathrm{Au})$ exhibits high oxidation resistance suitable for fabricating strain sensors $^{[49,50]}$. To realize its long-term utilization on the surface of human skin, e-skin must be extremely lightweight with imperceptible architectures and without any limitation and influence on body motion. It also requires skin-compatible interfaces with the targeted skin with a soft, curved dynamic surface in a stable, safe fashion that does not easily split away from the skin surface, as well as outstanding operational performance characteristics with high sensitivity and accuracy.

Herein, we report an ultrathin skin-integrated strain sensor based on the piezoresistive effect with excellent stability and repeatability. The key component is the Au electrode in the format of a fractal curve that is sensitive to ambient strain variations. To minimize the effect of the device on the skin strain field distribution, the overall dimensions of the sensor are $5 \mathrm{~mm} \times 2 \mathrm{~mm} \times 6.2 \mu \mathrm{m}$ (length $\times$ width $\times$ thickness) with a mass of $0.204 \mathrm{mg}$. Compared with conventional strain sensors based on the piezoresistive effect, our strain sensor demonstrates significantly reduced dimensions with a competitive stretching range and sensitivity [Supplementary Table 1] ${ }^{[51-56]}$. Guided by theoretical modeling, the advanced structural design enables conformal integration with the skin under large mechanical deformations. In order to yield the intimate contact on complex textures of epidermal surfaces, a liquid bandage is sprayed as a connection layer between the sensors and the epidermal surface. Compared to the commonly adopted PDMS layer, the liquid bandage possesses the advantages of low thickness $(1 \mu \mathrm{m})$ and lightweight. The tight bondage between the sensors and the epidermal surface allows the users' unhindered movements and providing 
precise measurements of the body motions sensing in a highly accurate approach. By accurately capturing finger motions and pulse rate, the sensor provides a promising alternative for real-time monitoring human health. In addition, with signals obtained from the strain sensors, a six-degree-of-freedom prosthetic arm is then used to imitate the motion of the human hand, by applying to program for sensitivity and detection adjustment.

\section{RESULTS AND DISCUSSION}

Figure 1A presents a schematic of the strain sensor. To ensure the surface smoothness and the fabrication compatibility, a quartz glass was first used as the substrate, with a spin coated poly(methyl methacrylate) (PMMA) thin film on top as a sacrificial layer and a thin polyimide (PI) film as the metal electrode supporting layer. Metallic layers $(\mathrm{Cr} / \mathrm{Au})$ were deposited on the PI film and patterned by photolithography, serving as the functional electrode for strain change detection. Spin-casting another PI film and selectively dry etching by reactive ion etching provided a top encapsulation layer for building a robust system. Dissolving the PMMA with acetone enabled transfer printing of the electrode onto the targeted skin area with pre-sprayed liquid bandage through a water-soluble tape (WST). Finally, an additional layer of liquid bandage was sprayed on top of the electronics to encapsulate the exposed gold electrode. The device features by the filamentary fractal design of the gold electrode $(200 \mathrm{~nm})$ and an ultrathin and stretchable encapsulation layer (liquid bandage, $1 \mu \mathrm{m}$ ) to enhance the overall flexibility and biaxial stretchability of the system [Figure 1B].

The liquid bandage acts as a tight adhesive and robust encapsulation for the strain sensor. A sensor is mounted on a volunteer's forearm over $12 \mathrm{~h}$ and during this period, the volunteer is allowed to perform all daily movements, such as walking, running and twisting his forearm. After $12 \mathrm{~h}$, the sensor is still mounted on their forearm with no evidence of flaking. According to the experimental studies, the ultrathin encapsulation layers can provide an excellent protection of the gold electrode underneath and the sensor could endure various mechanical forces, like continuous rubbing, while remaining undamaged [Supplementary Movie 1]. Apart from the external loads, the liquid bandage is waterproof, which could protect the sensor in moisture environments. There is experimental study proves that, with the encapsulation of the liquid bandage, the sensor is approximately unaffected in a watery environment, where the change in the electrical signal $\left(\Delta \mathrm{R} / \mathrm{R}_{0}\right)$ is less than $\pm 0.02 \%$ [Supplementary Figure 1]. This tight adhesion and waterproof capability of the liquid bandage allow the strain sensor to work unaffected under large body movements and a sweated skin surface.

The overall dimensions of the strain sensor are $5 \mathrm{~mm} \times 2 \mathrm{~mm} \times 6.2 \mu \mathrm{m}$ (length $\times$ width $\times$ thickness), thereby making it convenient and comfortable when attached to the human body, as shown in Figure $1 \mathrm{C}$. Compared with recently reported piezoresistive strain sensors, our device demonstrates superior dimensions with a competitive stretching range and sensitivity, as shown in Supplementary Table 1. The ultrathin design combined with filamentary fractal Au wires allows the device to be stretched, bent and twisted for the seamless and conformal integration onto curvilinear surfaces of human epidermis [Figure 1D and E]. It is found that most of the skin surface would stretch within $20 \%$, without any external stretching [Supplementary Table 2], indicating that the strain sensor could be mounted on most of the body parts for the body movement monitoring. Finite element analysis shows that the maximum equivalent strain in $\mathrm{Au}$ is less than a yield strain of $0.3 \%$ for the device under $280^{\circ}$ of bending (bending radius of $\sim 2.7 \mathrm{~mm}$ ), $20 \%$ stretching and $90^{\circ}$ of twisting [Figure 1D]. These results highlight that this robust and stretchable device can operate under realistic physiological loads. 
A
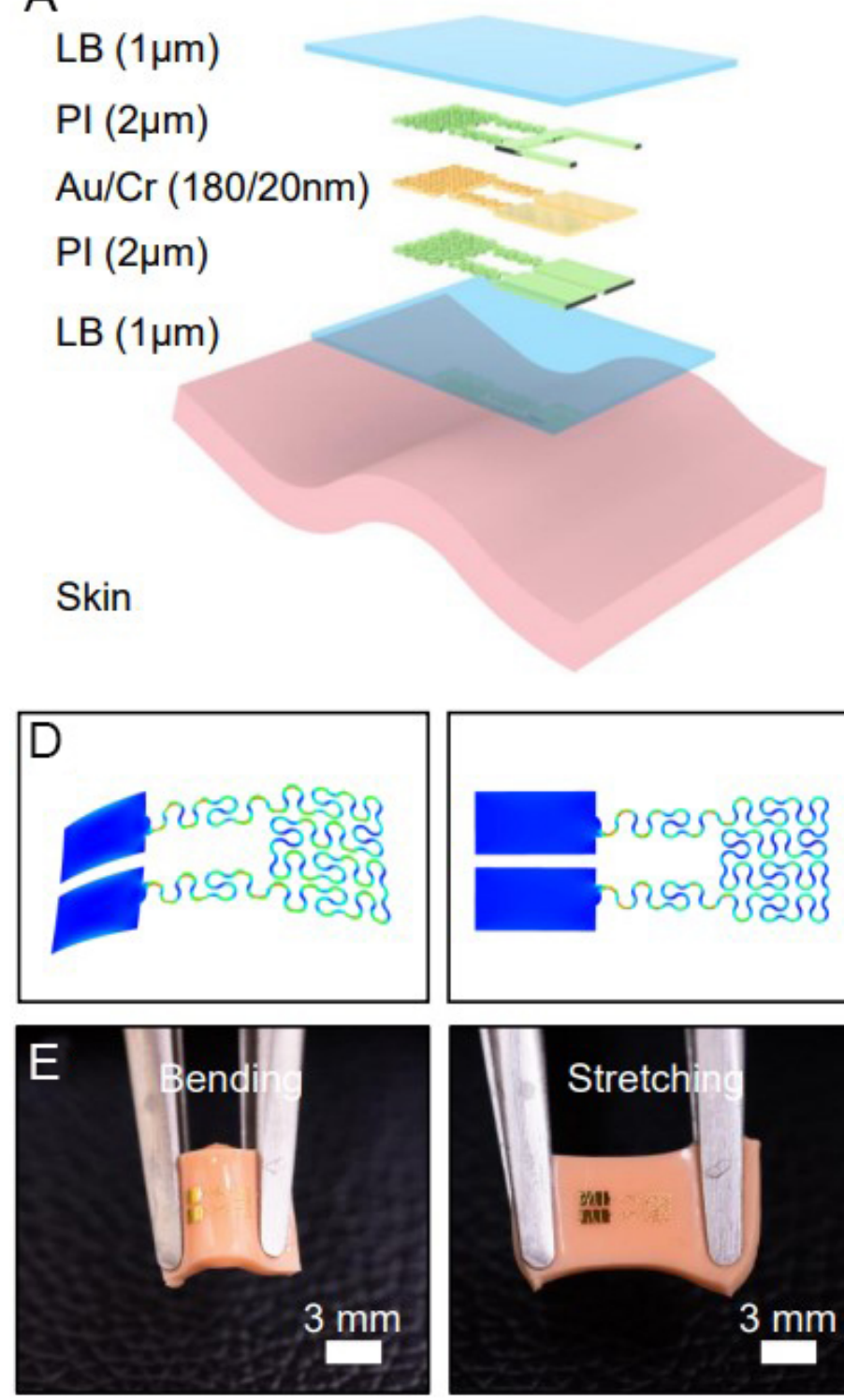
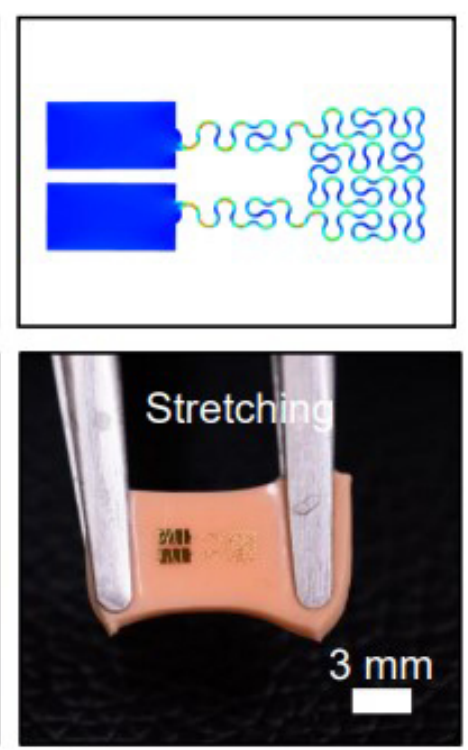
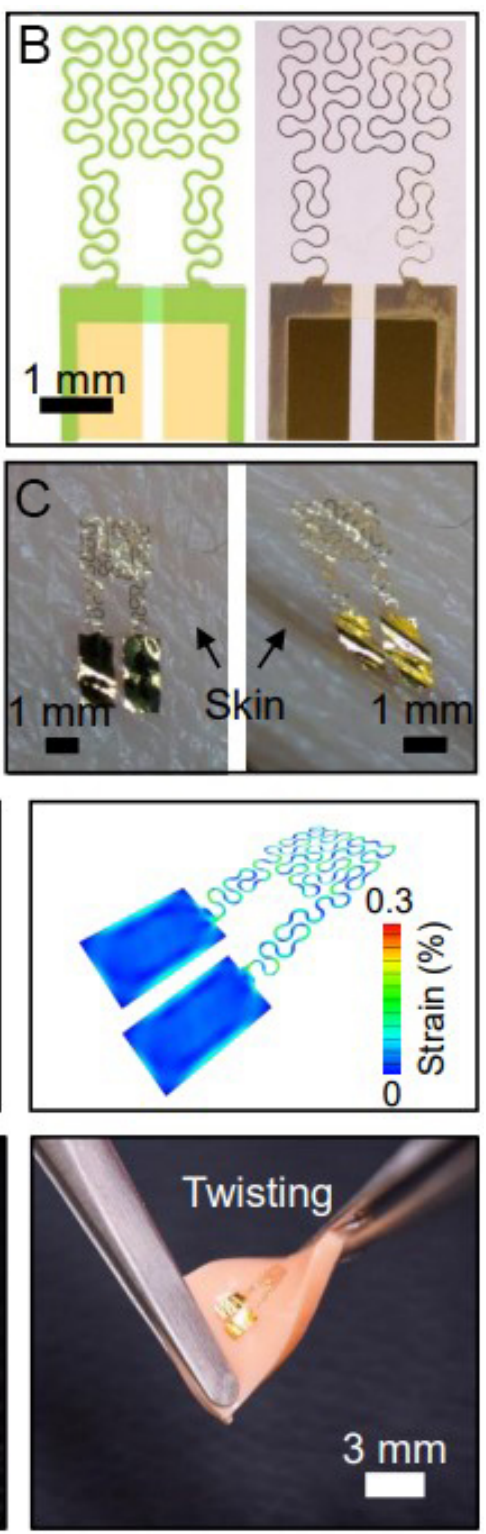

Figure 1. Flexible, ultrathin, skin-integrated and Au-based strain sensor. (A) Schematic of strain sensor. (B) Optical image of patterned $\mathrm{Au} / \mathrm{Cr}$ electrode design. (C) Optical image of strain sensor mounted on the human epidermal surface. (D) Finite element analysis of strain distribution on strain sensor under stretching, twisting and bending. (E) Optical image of strain sensor attached on a pink PDMS surface with three mechanical deformations, including bending, stretching and twisting.

Figure $2 \mathrm{~A}$ and $\mathrm{B}$ present the electrical signal $\left(\Delta R / R_{0}\right)$ as a function of strain variation under the static state along length and width, respectively. It is obvious that the electrical response increases linearly with increasing strain along the two directions. At the strain change of $20 \%$, the values of $\Delta R / R_{0}$ can reach $0.76 \%$ and $0.61 \%$ under longitudinal and transverse tensions, respectively. It is proved that the strain sensor is sensitive to both longitudinal and transverse strain and it is able to function at $20 \%$ strain in either direction. To investigate the frequency effect on the electrical signal, the device was tested under a constant strain variation (12.5\%) with different frequencies from 1 to $6 \mathrm{~Hz}$, fully covering the frequencies triggered by human daily motions, as shown in Figure $2 \mathrm{C}$. It is found that the low frequency has no effect on the electrical response of the device due to its fast response time (0.04 s) [Figure 2D]. To demonstrate the high durability of the strain sensor, fatigue tests were conducted with repeated stretching for 150 cycles at $1 \mathrm{~Hz}$ 

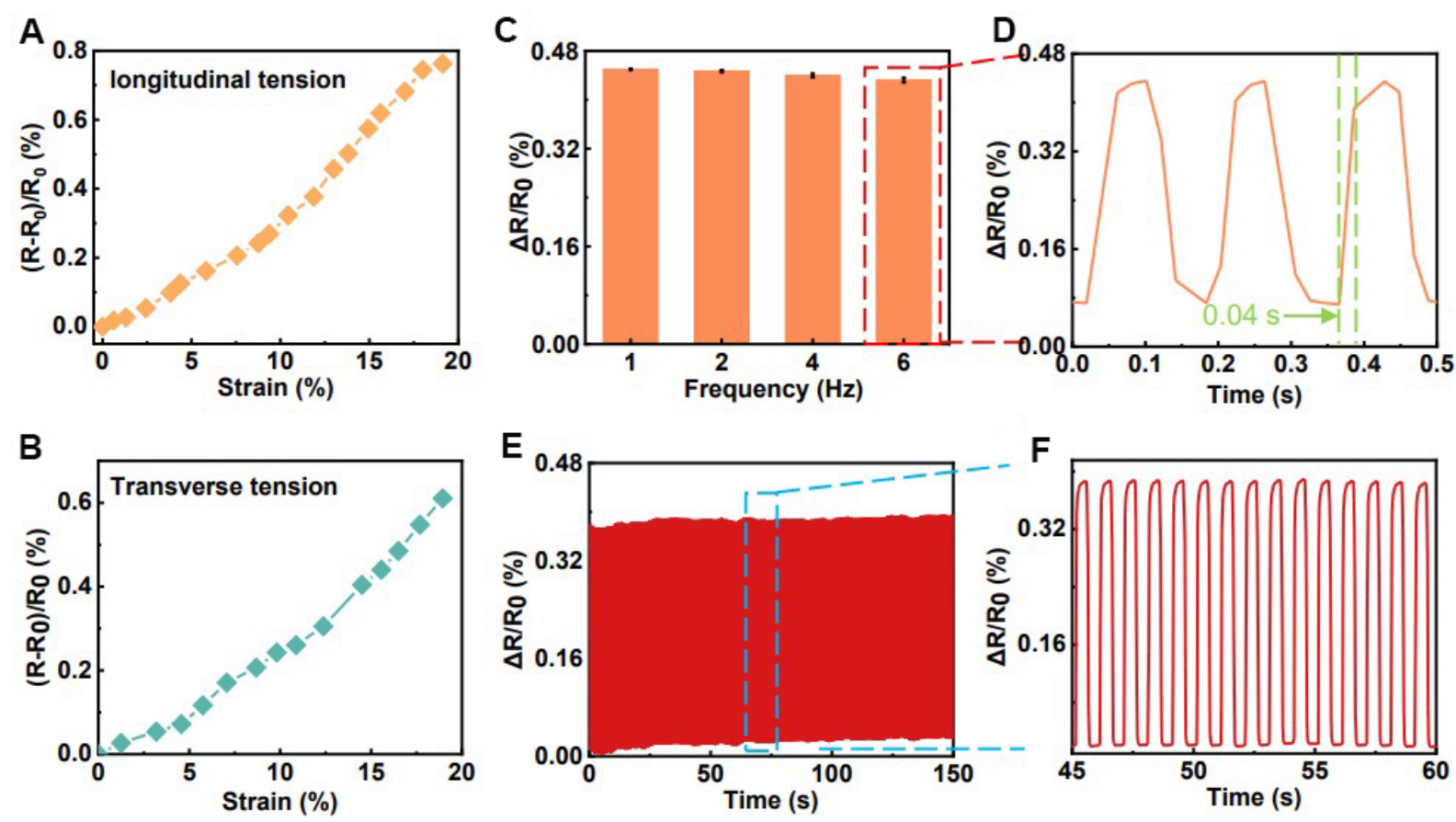

Figure 2. Electrical characteristics of strain sensor. (A) Electrical signals $\left(\Delta R / R_{0}\right)$ of strain sensor at different longitudinal tensions. (B) Electrical signals $\left(\Delta R / R_{0}\right)$ of strain sensor at different transverse tensions. (C, D) Electrical signals $\left(\Delta R / R_{0}\right)$ of strain sensor at different frequencies under $12.5 \%$ strain and its detailed signals at $6 \mathrm{~Hz}$. $(E, F)$ Electrical signals $\left(\Delta R / R_{0}\right)$ of strain sensor in a fatigue test for 150 cycles and its detailed signals from a selected range.

under $11.8 \%$ strain [Figure 2E] with electrical signals ranging from $0 \%$ to $0.4 \%$ [Figure $2 \mathrm{~F}$ ]. The results prove that the strain sensor is sufficiently durable to endure highly intensive stretching and maintain stable electrical signal outputs.

To further demonstrate its meticulous structure, the sensor is mounted on the back of a hand [Figure 3A]. Due to its ultrathin thickness and good adhesion of the encapsulation layer, the device can be conformally mounted onto human skin without any delamination during stretching. As shown in Figure $3 \mathrm{~B}$, the electrical signals $\left(\Delta \mathrm{R} / \mathrm{R}_{0}\right)$ at bending angles of $38^{\circ}, 61^{\circ}$ and $90^{\circ}$ are $0.121 \%, 0.285 \%$ and $0.567 \%$, respectively. This proves that the strain sensor is capable of capturing small body movements and output obvious signals, which are later applied in robotics control. As shown in Figure $3 \mathrm{C}$ and D, a sensor is attached onto an experimenter's wrist for pulse rate recording (given with its circuit diagram), where the measured relative voltage shows a regular variation ranging from $0 \%$ to $0.073 \%$, along with the pulse rate. It is sensitive towards the human pulse, which demonstrates the potential of the flexible skin-integrated sensors in the fields of clinical application. In Figure 3E, it shows an optical image of the strain sensor mounted on a volunteer's ankle for his walking motion detection. The $\Delta \mathrm{R} / \mathrm{R}_{0}$ varies along with the paces, ranging from $0.0173 \%$ to $0.354 \%$ [Figure $3 \mathrm{~F}$ ], which shows its capability of human motion capturing. Its sensitivity toward muscle contraction could be possibly applied in the field of motion detection in sports.

Next, the strain sensor is applied for human-machine interfaces by demonstrating the robotic hand control. The schematic of the testing circuit is shown in Figure 4A, in which the control system is composted with six strain sensors and their corresponding amplifiers (INA828, Texas Instruments) and an Arduino board. Figure $4 \mathrm{~B}$ and $\mathrm{C}$ show the optical images of the strain sensors mounted on the joints of a hand and circuits connected with the Arduino board (Arduino nano with ATMEGA328P-MU chip), respectively. Those 

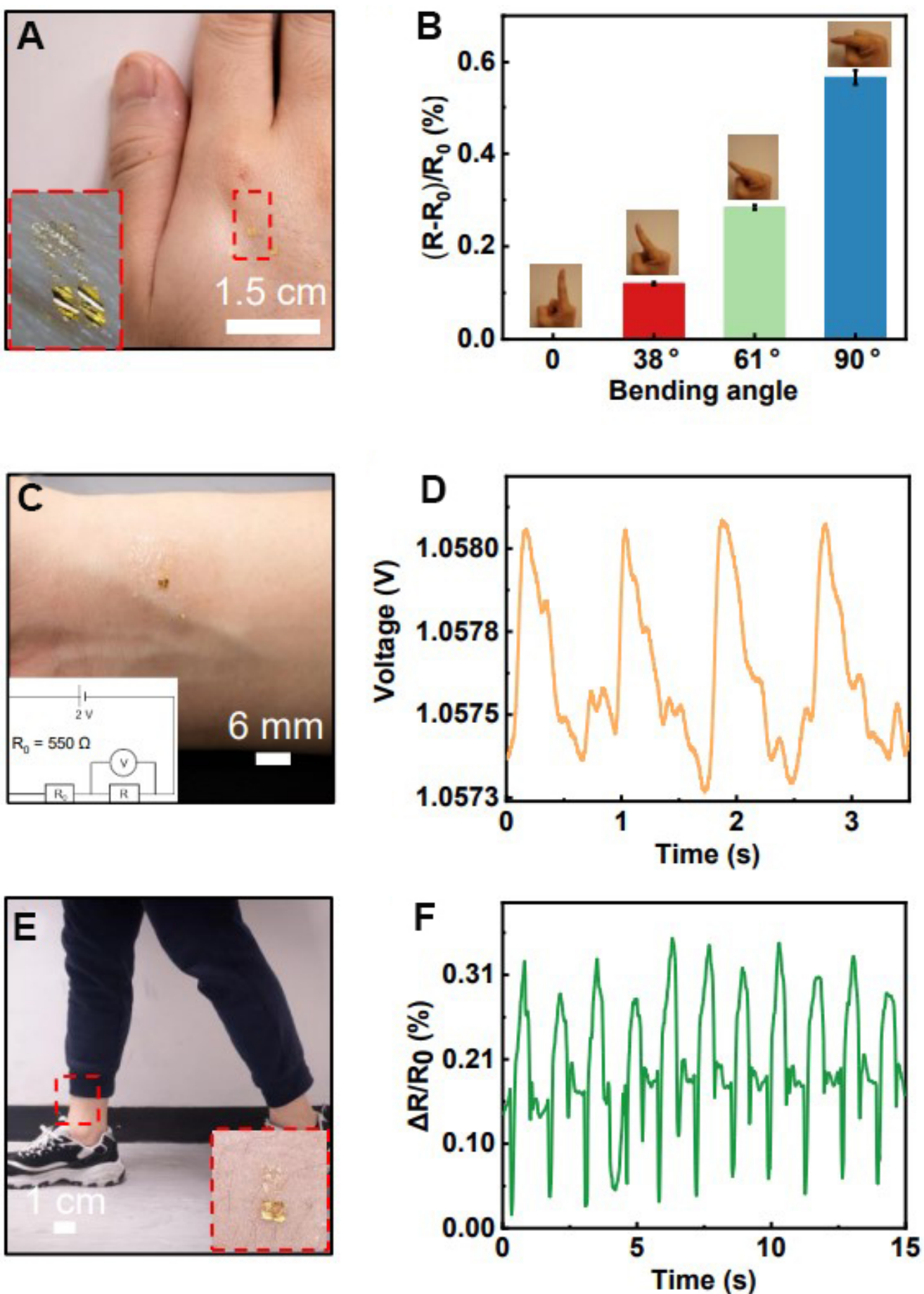

Figure 3. Electrical signals of flexible strain sensor under different external stimuli. (A) Optical image of strain sensor mounted on the back of a hand and its enlarged optical image of the strain sensor during deformation. (B) Electrical signals $\left(\Delta R / R_{0}\right)$ of strain sensor under different finger bending angles. (C) Optical image of strain sensor mounted on a wrist for human pulse rate detection and its electrical diagram. (D) Electrical signals (V) of strain sensor in the pulse rate test in (C). (E) Optical image of strain sensor mounted on a waist for ankle for walking motion detection. $(F)$ Electrical signals $\left(\Delta R / R_{0}\right)$ of strain sensor under the walking motion test.

sensors are mounted on the joints of the hand to capture the finger motions, which lead to a smooth control of the robotic hand. It is able to imitate the gestures of the hand almost without any detention. In Figure 4D, 

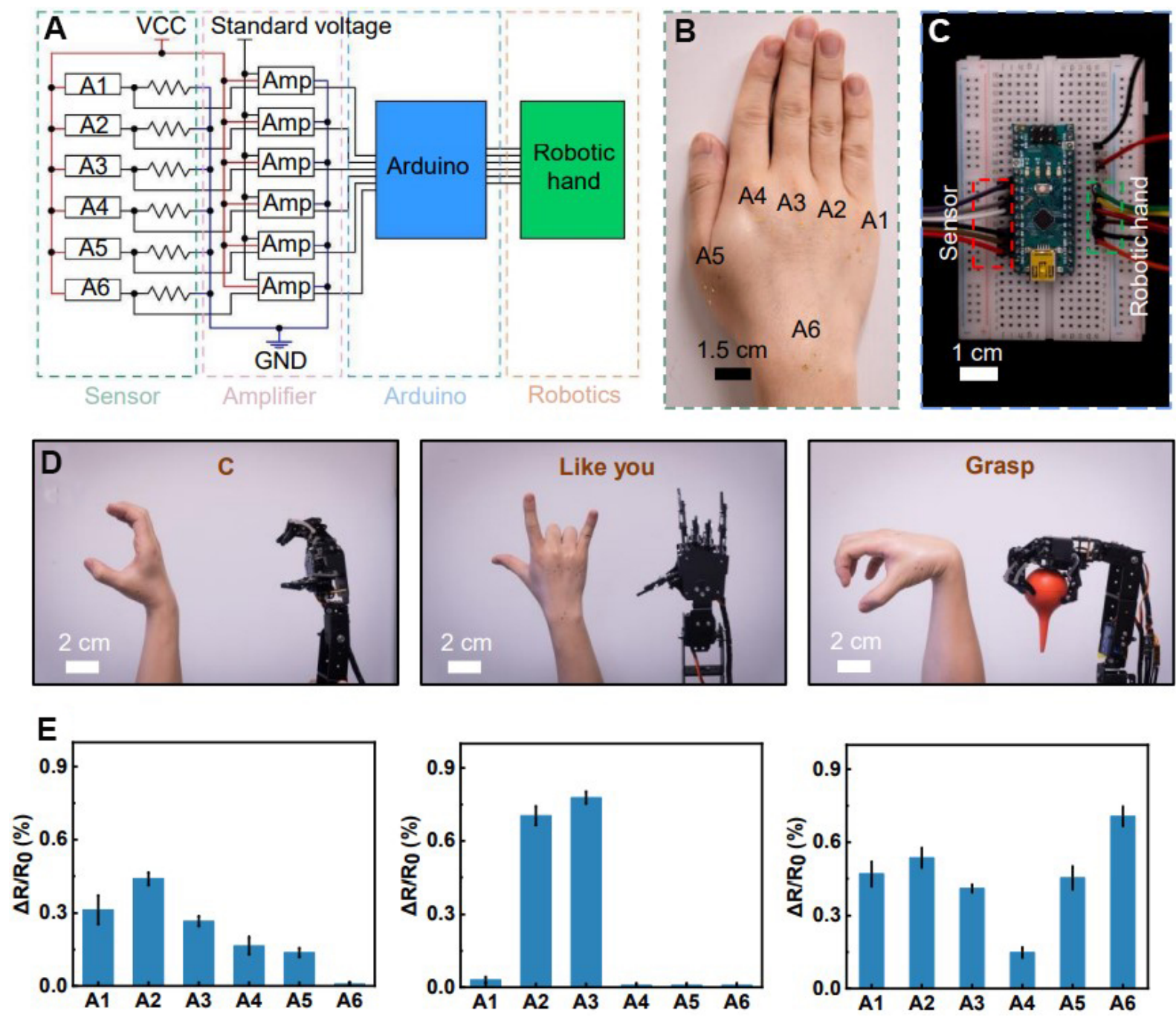

Figure 4. Robotic hand controlling performed by flexible strain sensors. (A) Schematic diagram of testing circuit for controlling robotic hand. (B) Optical image of six sensors mounted on the joints of a hand for robotic hand control. (C) Optical image of Arduino breadboard linked with the sensors and the robotic hand. (D) Optical image illustrating that the sensors control the robotic hand to make gestures of " $\mathrm{C}$ ", "like you" and "grasp" and their corresponding electrical signals ( $R / R_{0}$ ) of sensors at different gestures (E).

some meaningful hand gestures, including "C", "like you", and "grasp", are demonstrated, which proves the high feasibility of robotic hand control. Moreover, there are three corresponding graphs underneath illustrating the electrical responses of the sensors for different hand gestures. This demonstrates that the signal intensity varies with the hand's bending degree that at the fingers' complete bending and straightening conditions, the electrical signals $\left(\Delta R / R_{0}\right)$ are $0.75 \%$ and $0.04 \%$, respectively [Figure $4 \mathrm{E}$ ]. This distinct disparity of the electrical signals leads to the accurate control of the robotic hand. To summarize, we have demonstrated a human-machine interface application of the strain sensor by controlling a robotic hand in a natural and smooth approach, thereby indicating the high feasibility of applying flexible strain sensors in human-machine interfaces.

\section{CONCLUSION}

An ultrathin Au-based stretchable strain sensor has been developed for body motion caption and humanmachine interfaces. It possesses the advantages of miniaturized dimensions $(5 \mathrm{~mm} \times 2 \mathrm{~mm} \times 6.2 \mu \mathrm{m})$, skin 
integrability, high stability and stretchability, which allow it to be tightly mounted on the epidermal surface for body motion capturing. Its electrical signal output $\Delta \mathrm{R} / \mathrm{R}_{0}$ can rise to $0.76 \%$ and $0.61 \%$ under $20 \%$ longitudinal and transverse stretching rate, respectively, and it can undergo repeated stretching for 150 cycles at $1 \mathrm{~Hz}$ under $11.8 \%$ strain. With a liquid bandage as the encapsulation, the strain sensor can be tightly mounted on epidermal surface that can measure the human pulse rate and the walking motions. Furthermore, the strain sensors are applied in robotic hand control, which can imitate the finger motions of the human hand precisely, smoothly, and almost without any detention. The results in this work present the potential in body motion measuring and robotic control, which indicates a new strategy for flexible sensors in clinical applications and human-machine interfaces.

\section{EXPERIMENTAL}

\section{Assembly of tattoo-like e-skin}

First, a quartz glass was cleaned with acetone, ethanol and deionized water sequentially. Then, a PMMA solution $(20 \mathrm{mg} / \mathrm{mL})$ was spin-coated onto the surface of the cleaned glass at $2000 \mathrm{rpm}$ for $30 \mathrm{~s}$ and then baked on a hotplate at $200 \mathrm{C}$ for $20 \mathrm{~min}$. The PMMA thin film served as a sacrificial layer. Afterwards, polyamic acid solution $(12.0 \mathrm{wt} . \% \pm 0.5 \mathrm{wt} . \%, 3 \mu \mathrm{m})$ was spin-coated on the PMMA sacrificial layer at 3000 $\mathrm{rpm}$ for $30 \mathrm{~s}$. The PI thin film was then cured on the hotplate at $250{ }^{\circ} \mathrm{C}$ for $30 \mathrm{~min}$ to densify. $\mathrm{Cr}(40 \mathrm{~nm})$ and $\mathrm{Au}(200 \mathrm{~nm})$ were next deposited on the PI film by magnetron sputtering, patterned with photolithography and further wet etched to form the desired pattern. The photolithography process was carried out by exposing the pre-spin-coated $(3000 \mathrm{rpm}, 30 \mathrm{~s})$ and soft-baked $\left(110^{\circ} \mathrm{C}, 3 \mathrm{~min}\right)$ positive photoresist (PR, AZ 5214, AZ Electronic Materials) to ultraviolet light for $5 \mathrm{~s}$. The pattern was then developed in an AZ 300MIF developer for $15 \mathrm{~s}$, followed by a post bake at $110 \mathrm{C}$ for $3 \mathrm{~min}$.

After etching the $\mathrm{Au} / \mathrm{Cr}$ layer, the PR was removed with acetone and rinsed with deionized water. A second layer of PI thin film $(2 \mu \mathrm{m})$ was then spin coated $(3000 \mathrm{rpm}, 30 \mathrm{~s})$ and annealed $\left(250{ }^{\circ} \mathrm{C}\right.$, $\left.30 \mathrm{~min}\right)$, followed by selectively dry etching (Oxford Plasma-Therm 790 RIE system, $200 \mathrm{~W}, 10 \mathrm{~min}$ ). The patterned PI thin film served as the encapsulation layer covering the whole interconnects except the electrode areas. Next, the sample was immersed in acetone for $12 \mathrm{~h}$ to fully dissolve the PMMA layer. WSTs were utilized as a stamp to pick up the prepared pattern. An ultrathin layer of liquid bandage ( $1 \mu \mathrm{m}$, Banitore company) was sprayed on the target area of human skin. Tightly attaching the WSTs on the target area then dried in the air at $25^{\circ} \mathrm{C}$ for 5 min formed strong mechanical bonding. Immersing the sample in water to remove the WSTs and realize stretchable electrodes on human skin. Next, a liquid bandage layer $(1 \mu \mathrm{m})$ was sprayed on the top of the electrode. The liquid bandage was dried in air for $5 \mathrm{~min}$ to form a robust structure with strong interfacial bonding between different layers.

\section{Characterization}

The resistance was measured by a DAQ6510 data acquisition/multi-meter system. The tiny voltage variance in Figure 3D was calculated by measuring the voltage of a fixed value resistor connected in series with the triboelectric nanogenerator (the voltage was measured by PL3516/P Powerlab 16/35, which owns much lower noise signal and higher sampling rate than the DAQ6510 multimeter system).

\section{Mechanical simulations}

The finite element analysis commercial software ABAQUS (Analysis User's Manual 2016) was utilized to design the layout of strain sensor and study the corresponding mechanical performance. The objective was to decrease the strain level in Au layer under different typical loads (stretching, bending and twisting). The phantom skin was modeled by hexahedron elements (C3D8R), while the thin $\mathrm{Au}, \mathrm{Cr}$, liquid bandage and PI layers was modeled by shell elements $(\mathrm{S} 4 \mathrm{R})$. The minimal element size was one quarter of the width of the Au wires $(50 \mu \mathrm{m})$, which ensured the convergence of the mesh and the accuracy of the simulation results. 
The elastic modulus $(\mathrm{E})$ and Poisson's ratio $(v)$ used in the analysis were $\mathrm{EAu}=78 \mathrm{GPa}, v \mathrm{Au}=0.41$; EPI $=$ 2.5 GPa, vPI = 0.34; Eskin $=60 \mathrm{kPa}$, vskin $=0.5 ; \mathrm{EGr}=294 \mathrm{GPa}, \mathrm{vGr}=0.21$ and $\mathrm{ELB}=85 \mathrm{MPa}, \mathrm{vLB}=0.42$.

\section{DECLARATIONS}

\section{Authors' contributions}

Data curation, formal analysis, investigation, methodology, writing original draft: Wong TH

Conceptualization: Yiu CK, Zhou J

Methodology: Liu Y, Zhao L, Yoo W, Park W

Visualization: Yao K

Software and data analysis: Song Z, Xie Z

Data curation, formal analysis and supervision: Song $\mathrm{E}$

Conceptualization, funding acquisition, project administration, resources, supervision and writing, review and editing: Yu X

\section{Financial support and sponsorship}

This work was supported by City University of Hong Kong (Grants No. 9610423, 9667199, 9667221, 9680322), Research Grants Council of the Hong Kong Special Administrative Region (Grant No. 21210820, 11213721), Shenzhen Science and Technology Innovation Commission (Grant No. JCYJ20200109110201713) and Hong Kong Centre for Cerebro-Cardiovascular Health Engineering. Xie Z acknowledges the support from the National Natural Science Foundation of China (Grant No. 12072057), LiaoNing Revitalization Talents Program (Grant No. XLYC2007196), and Fundamental Research Funds for the Central Universities (Grant No. DUT20RC(3)032). Song E acknowledges the support from Shanghai Municipal Science and Technology Major Project (No.2018SHZDZX01), ZJ Lab, and Shanghai Center for Brain Science and Brain-Inspired Technology.

\section{Conflicts of interest}

All authors declared that there are no conflicts of interest.

\section{Consent for publication}

Not applicable.

\section{Ethical approval and consent to participate}

Not applicable.

\section{Consent for publication}

Not applicable.

\section{Copyright}

(c) The Author(s) 2021.

\section{REFERENCES}

1. Yeo WH, Kim YS, Lee J, et al. Multifunctional epidermal electronics printed directly onto the skin. Adv Mater 2013;25:2773-8. DOI PubMed

2. Jeong JW, Yeo WH, Akhtar A, et al. Materials and optimized designs for human-machine interfaces via epidermal electronics. Adv Mater 2013;25:6839-46. DOI PubMed

3. Zhu Z, Li R, Pan T. Imperceptible epidermal-iontronic interface for wearable sensing. Adv Mater 2018;30:1705122. DOI PubMed

4. Liu Y, Norton JJ, Qazi R, et al. Epidermal mechano-acoustic sensing electronics for cardiovascular diagnostics and human-machine interfaces. Sci Adv 2016;2:e1601185. DOI PubMed PMC

5. Reeder JT, Choi J, Xue Y, et al. Waterproof, electronics-enabled, epidermal microfluidic devices for sweat collection, biomarker analysis, and thermography in aquatic settings. Sci Adv 2019;5:eaau6356. DOI PubMed PMC

6. Yu X, Xie Z, Yu Y, et al. Skin-integrated wireless haptic interfaces for virtual and augmented reality. Nature 2019;575:473-9. DOI 
PubMed

7. Yeo JC, Lim CT. Emerging flexible and wearable physical sensing platforms for healthcare and biomedical applications. Microsyst Nanoeng 2016;2:16043. DOI PubMed PMC

8. Liu Y, Zhao L, Avila R, et al. Epidermal electronics for respiration monitoring via thermo-sensitive measuring. Materials Today Physics 2020;13:100199. DOI

9. Kim CH, Lee DH, Youn J, Lee H, Jeong J. Simple and cost-effective microfabrication of flexible and stretchable electronics for wearable multi-functional electrophysiological monitoring. Sci Rep 2021;11:14823. DOI PubMed PMC

10. Guo J, Yu Y, Zhang D, Zhang H, Zhao Y. Morphological hydrogel microfibers with MXene encapsulation for electronic skin. Research (Wash D C) 2021;2021:7065907. DOI PubMed PMC

11. Liu Y, Zhao L, Wang L, et al. Skin-integrated graphene-embedded lead zirconate titanate rubber for energy harvesting and mechanical sensing. Adv Mater Technol 2019;4:1900744. DOI

12. Tzou H, Tseng C. Distributed piezoelectric sensor/actuator design for dynamic measurement/control of distributed parameter systems: a piezoelectric finite element approach. J Sound Vib 1990;138:17-34. DOI

13. Ng TH, Liao WH. Sensitivity analysis and energy harvesting for a self-powered piezoelectric sensor. J Intell Mater Syst Struct 2005;16:785-97. DOI

14. Liu Y, Zheng H, Zhao L, et al. Electronic skin from high-throughput fabrication of intrinsically stretchable lead zirconate titanate elastomer. Research (Wash D C) 2020;2020:1085417. DOI PubMed PMC

15. Wang X, Zhang H, Dong L, et al. Self-powered high-resolution and pressure-sensitive triboelectric sensor matrix for real-time tactile mapping. Adv Mater 2016;28:2896-903. DOI PubMed

16. An T, Anaya DV, Gong S, et al. Self-powered gold nanowire tattoo triboelectric sensors for soft wearable human-machine interface. Nano Energy 2020;77:105295. DOI

17. Wu M, Gao Z, Yao K, et al. Thin, soft, skin-integrated foam-based triboelectric nanogenerators for tactile sensing and energy harvesting. Materials Today Energy 2021;20:100657. DOI

18. Yiu C, Wong TH, Liu Y, et al. Skin-like strain sensors enabled by elastomer composites for human-machine interfaces. Coatings 2020;10:711. DOI

19. Yan C, Wang J, Kang W, et al. Highly stretchable piezoresistive graphene-nanocellulose nanopaper for strain sensors. Adv Mater 2014;26:2022-7. DOI PubMed

20. Pacelli M, Caldani L, Paradiso R. Textile piezoresistive sensors for biomechanical variables monitoring. Conf Proc IEEE Eng Med Biol Soc 2006;2006:5358-61. DOI PubMed

21. Hu N, Fukunaga H, Atobe S, Liu Y, Li J. Piezoresistive strain sensors made from carbon nanotubes based polymer nanocomposites. Sensors (Basel) 2011;11:10691-723. DOI PubMed PMC

22. Zhu J, Zhou C, Zhang M. Recent progress in flexible tactile sensor systems: from design to application. Soft Sci 2021;1:3. DOI

23. Liu Y, Wang L, Zhao L, Yu X, Zi Y. Recent progress on flexible nanogenerators toward self-powered systems. InfoMat 2020;2:31840. DOI

24. Wang L, Liu Y, Liu Q, et al. A metal-electrode-free, fully integrated, soft triboelectric sensor array for self-powered tactile sensing. Microsyst Nanoeng 2020;6:59. DOI PubMed PMC

25. Zhou Y, He J, Wang H, et al. Highly sensitive, self-powered and wearable electronic skin based on pressure-sensitive nanofiber woven fabric sensor. Sci Rep 2017;7:12949. DOI PubMed PMC

26. Lou Z, Li, Wang L, Shen G. Recent progress of self-powered sensing systems for wearable electronics. Small 2017;13:1701791. DOI PubMed

27. Wang Y, Wang Y, Yang Y. Graphene-polymer nanocomposite-based redox-induced electricity for flexible self-powered strain sensors. Adv Energy Mater 2018;8:1800961. DOI

28. Lu Y, Biswas MC, Guo Z, Jeon JW, Wujcik EK. Recent developments in bio-monitoring via advanced polymer nanocomposite-based wearable strain sensors. Biosens Bioelectron 2019;123:167-77. DOI PubMed

29. Wang X, Zhang Y, Zhang X, et al. A highly stretchable transparent self-powered triboelectric tactile sensor with metallized nanofibers for wearable electronics. Adv Mater 2018;30:e1706738. DOI PubMed

30. Zhao G, Zhang Y, Shi N, et al. Transparent and stretchable triboelectric nanogenerator for self-powered tactile sensing. Nano Energy 2019;59:302-10. DOI

31. Yang M, Liu J, Liu D, et al. A Fully Self-healing piezoelectric nanogenerator for self-powered pressure sensing electronic skin. Research (Wash D C) 2021;2021:9793458. DOI PubMed PMC

32. Shu YC, Lien IC. Analysis of power output for piezoelectric energy harvesting systems. Smart Mater Struct 2006;15:1499-512. DOI

33. Feng W, Zheng W, Gao F, et al. Sensitive electronic-skin strain sensor array based on the patterned two-dimensional $\alpha$ - $\mathrm{In}_{2} \mathrm{Se}_{3}$. Chem Mater 2016;28:4278-83. DOI

34. Park J, Lee Y, Hong J, et al. Tactile-direction-sensitive and stretchable electronic skins based on human-skin-inspired interlocked microstructures. ACS Nano 2014;8:12020-9. DOI PubMed

35. Lipomi DJ, Vosgueritchian M, Tee BC, et al. Skin-like pressure and strain sensors based on transparent elastic films of carbon nanotubes. Nat Nanotechnol 2011;6:788-92. DOI PubMed

36. Cheng Y, Wang R, Zhai H, Sun J. Stretchable electronic skin based on silver nanowire composite fiber electrodes for sensing pressure, proximity, and multidirectional strain. Nanoscale 2017;9:3834-42. DOI PubMed

37. Kanao K, Harada S, Yamamoto Y, et al. Highly selective flexible tactile strain and temperature sensors against substrate bending for an artificial skin. RSC Adv 2015;5:30170-4. DOI 
38. Gong S, Lai DTH, Su B, et al. Highly stretchy black gold e-skin nanopatches as highly sensitive wearable biomedical sensors. Adv Electron Mater 2015;1:1400063. DOI

39. Luo N, Huang Y, Liu J, Chen SC, Wong CP, Zhao N. Hollow-structured graphene-silicone-composite-based piezoresistive sensors: decoupled property tuning and bending reliability. Adv Mater 2017;29:1702675. DOI PubMed

40. Chen H, Miao L, Su Z, et al. Fingertip-inspired electronic skin based on triboelectric sliding sensing and porous piezoresistive pressure detection. Nano Energy 2017;40:65-72. DOI

41. Niu D, Jiang W, Ye G, et al. Graphene-elastomer nanocomposites based flexible piezoresistive sensors for strain and pressure detection. Materials Research Bulletin 2018;102:92-9. DOI

42. Canavese G, Lombardi M, Stassi S, Pirri CF. Comprehensive characterization of large piezoresistive variation of Ni-PDMS composites. AMM 2011;110-116:1336-44. DOI

43. Cai G, Wang J, Qian K, Chen J, Li S, Lee PS. Extremely stretchable strain sensors based on conductive self-healing dynamic crosslinks hydrogels for human-motion detection. Adv Sci (Weinh) 2017;4:1600190. DOI PubMed PMC

44. Jing X, Mi H, Peng X, Turng L. Biocompatible, self-healing, highly stretchable polyacrylic acid/reduced graphene oxide nanocomposite hydrogel sensors via mussel-inspired chemistry. Carbon 2018;136:63-72. DOI

45. Liu S, Li L. Ultrastretchable and self-healing double-network hydrogel for 3D printing and strain sensor. ACS Appl Mater Interfaces 2017;9:26429-37. DOI PubMed

46. Yin F, Yang J, Peng H, Yuan W. Flexible and highly sensitive artificial electronic skin based on graphene/polyamide interlocking fabric. J Mater Chem C 2018;6:6840-6. DOI

47. Zhao L, Qiang F, Dai SW, et al. Construction of sandwich-like porous structure of graphene-coated foam composites for ultrasensitive and flexible pressure sensors. Nanoscale 2019;11:10229-38. DOI PubMed

48. Nie P, Wang R, Xu X, et al. High-performance piezoresistive electronic skin with bionic hierarchical microstructure and microcracks. ACS Appl Mater Interfaces 2017;9:14911-9. DOI PubMed

49. Hsu MS, Chen YL, Lee CY, Chiu HT. Gold nanostructures on flexible substrates as electrochemical dopamine sensors. ACS Appl Mater Interfaces 2012;4:5570-5. DOI PubMed

50. Zhu Y, Moran-mirabal J. Highly bendable and stretchable electrodes based on micro/nanostructured gold films for flexible sensors and electronics. Adv Electron Mater 2016;2:1500345. DOI

51. Li YQ, Huang P, Zhu WB, Fu SY, Hu N, Liao K. Flexible wire-shaped strain sensor from cotton thread for human health and motion detection. Sci Rep 2017;7:45013. DOI PubMed PMC

52. Sankar V, Nambi A, Bhat VN, et al. Waterproof flexible polymer-functionalized graphene-based piezoresistive strain sensor for structural health monitoring and wearable devices. ACS Omega 2020;5:12682-91. DOI PubMed PMC

53. Wang X, Li J, Song H, Huang H, Gou J. Highly Stretchable And Wearable Strain Sensor Based On Printable Carbon Nanotube Layers/Polydimethylsiloxane Composites With Adjustable Sensitivity. ACS Appl Mater Interfaces 2018;10:7371-80. DOI PubMed

54. Zheng Y, Li Y, Zhou Y, et al. High-performance wearable strain sensor based on graphene/cotton fabric with high durability and low detection limit. ACS Appl Mater Interfaces 2020;12:1474-85. DOI PubMed

55. Zheng S, Wu X, Huang Y, et al. Highly sensitive and multifunctional piezoresistive sensor based on polyaniline foam for wearable Human-Activity monitoring. Composites Part A: Applied Science and Manufacturing 2019;121:510-6. DOI

56. Zhang C, Zhou G, Rao W, Fan L, Xu W, Xu J. A simple method of fabricating nickel-coated cotton fabrics for wearable strain sensor. Cellulose 2018;25:4859-70. DOI 